\title{
A NOTE ON DOUBLY TRANSITIVE GROUPS
}

\author{
PETER LORIMER
}

(Received 22 November 1965)

W. Feit [1], N. Itô [2] and M. Suzuki [3] have determined all doubly transitive groups with the property that only the identity fixes three symbols. It is of interest to the theory of projective planes to determine whether any of these groups contain a sharply doubly transitive subset (see Definition 1). It is found that if such a group $G$ contains such a subset $R$ then $R$ is a normal subgroup of $G$, i.e. $R$ is a doubly transitive normal subgroup of $G$ in which only the identity fixes two symbols.

Definition 1. If $R$ is a set of permutations on a set $\Sigma$ of $n$ symbols, then $R$ is said to be sharply doubly transitive on $\Sigma$ if the identity 1 is a member of $R, R$ contains $n(n-1)$ elements and $R$ is doubly transitive on $\Sigma$.

It should be noted that the definition implies that if $(a, b)$ and $(c, d)$ are two pairs of symbols of $\Sigma$ then there is exactly one $r \in R$ with the properties $r(a)=c, r(b)=d$.

In the sequel, $G$ is to be a doubly transitive group on a set $\Sigma$ of $n$ elements, only the identity of $G$ is to fix three symbols of $\Sigma$ and $G$ is to contain a sharply doubly transitive subset $R$.

It is clear that the subset $K_{a}$ of $R$ which consists of those elements which fix $a \in \Sigma$ contains $n-1$ elements. Also the subset $R^{*}$ of $R$ which consists of those elements of $R$ which fix no symbol of $\Sigma$ together with the identity contains $n$ elements.

Let $G_{a}$ be the subgroup of $G$ consisting of those elements of $G$ which fix the symbol $a \in \Sigma$.

Lemma 1. If $a \in \Sigma$, then $K_{a}$ is a subgroup of $G$ and consists of all those elements of $G$ which fix a alone, together with the identity.

Proof. Consider $G_{a}$ as a group of permutations on the set $\Sigma^{\prime}=\Sigma-\{a\}$. $G_{a}$ is transitive and only the identity fixes two symbols of $\Sigma^{\prime}$. Hence, by Frobenius' Theorem, $G_{a}$ contains $n-2$ elements which fix no symbol of $\Sigma^{\prime}$, and these, together with the identity, form a normal subgroup of $H_{a}$. It is clear that this subgroup consists of the elements of $K_{a}$.

LEMma 2. If $r_{1}, r_{2} \in R^{*}$, then either $r_{1}=r_{2}$ or $r_{1}^{-1} r_{2}$ fixes no symbol of $\Sigma$. 
LEMMA 3. If $r_{1}, r_{2} \in R$, then either $r_{1}=r_{2}$ or both $r_{1}^{-1} r_{2}$ and $r_{1} r_{2}^{-1}$ fix at most one symbol of $\Sigma$.

Lемма 4. Suppose $a, b \in \Sigma$ and $a \neq b$. If $k_{a} \in K_{a}$, then there exists exactly one $k_{b} \in K_{b}$ with the property: $k_{a} k_{b}$ fixes no symbol of $\Sigma$.

Proof. Suppose $c \in \Sigma, c \neq a, c \neq b$. Then there exists $\mathrm{k}_{b} \in K$ with the property $k_{a}^{-1}(c)=k_{b}(c)$. i.e. $k_{a} k_{b}(c)=c$. If $k_{a} k_{b}(c)=k_{a} k_{b}^{\prime}(c)=c$, then $k_{b}(c)=k_{b}^{\prime}(c)$ so that $k_{b}=k_{b}^{\prime}$. Hence there are $n-2$ elements $k_{b}$ of $K_{b}$ with the property $k_{a} k_{b}(c)=c$ for some $c$ in $\Sigma$.

$K_{b}$ contains $n-1$ elements which proves the theorem.

LEMMA 5. If $a \in \Sigma$ then $R=R^{*} K_{a}$.

ProOF. We show firstly that if $r_{1} k_{1}=r_{2} k_{2}, r_{i} \in R^{*}, k_{i} \in K_{a}$ then $r_{1}=r_{2}, k_{1}=k_{2}$.

If $r_{1} k_{1}=r_{2} k_{2}$, then $r_{1}^{-1} r_{2}=k_{2} k_{1}^{-1}$. Now $k_{2} k_{1}^{-1} \in K_{a}$ so that $r_{1}^{-1} r_{2}$ fixes a. Hence, by lemma $2, r_{1}=r_{2}$ and therefore $k_{1}=k_{2}$.

Hence each element of $R^{*} K_{a}$ is represented uniquely in the form $r k$, $r \in R^{*}, k \in K_{a}$, so that $R^{*} K_{a}$ contains $n(n-1)$ elements.

$R^{*} \subseteq R^{*} K_{a}$ as $1 \in K_{a}$.

If $b \in \Sigma$, it follows from lemma 3 that $R^{*} K_{a}$ contains $n-1$ elements fixing $b$ and no other symbol. Hence, by lemma $1, K_{b} \subseteq R^{*} K_{a}$.

Hence $R \subseteq R^{*} K_{a}$ and even $R=R^{*} K_{a}$ as both sets contain $n(n-1)$ elements.

LEMMA 6. If $a, b \in \Sigma$, then $K_{a} K_{b} \subseteq R$.

PROOF. If $k_{a} k_{b} \in K_{a} K_{b}$ and $k_{a} k_{b}$ fixes a symbol of $\Sigma$, then, by lemma $3, k_{a} k_{b}$ fixes exactly one symbol of $\Sigma$ so that, by lemma $1, k_{a} k_{b} \in K_{c}$ for some $c \in \Sigma$. Hence $k_{a} k_{b} \in R$.

Now, if $k_{a}$ is given, there exists unique $k_{b} \in K_{b}$ such that $k_{a} k_{b}$ fixes no symbol of $\Sigma$. But $R=R^{*} K_{b}$ so that there exists $h_{b} \in K_{b}$ and $r \in R^{*}$ with the property $k_{a}=r h_{b}^{-1}$. Then $r=k_{a} h_{b}$ so that, because of the uniqueness of $k_{b}$ we have $h_{b}=k_{b}$. Thus $k_{a} k_{b}=r \in R$.

Hence $K_{a} K_{b} \subseteq R$.

LEMMA 7. If $a \in \Sigma$, then $R K_{a}=R$.

Proof. $R=R^{*} K_{a}$ so that $R K_{a}=R^{*} K_{a} K_{a}=R^{*} K_{a}$ (as $K_{a}$ is a subgroup) $=R$.

LEMMA 8. If $a \neq b, r \in R, r \notin K_{a} K_{b}$, then $r^{-1} K_{a} r=K_{b}$.

PRoOF. If $r \in K_{a} K_{b}$, say $r=k_{a} k_{b}$, then $r^{-1} K_{a} r=k_{b}^{-1} K_{a} k_{b} \neq K_{b}$.

But $n-1$ elements $r$ of $R$ have the property $r^{-1} K_{a} r=K_{b}$ and $K_{a} K_{\imath}$ contains $(n-1)^{2}$ elements. The result follows. 
LEMMA 9. If $a, b, c$ are three different elements of $\Sigma$ and $r \in R$, then either $r \in K_{a} K_{b}$ or $r \in K_{a} K_{c}$.

PROOF. If $r \notin K_{a} K_{b}$ and $r \notin K_{a} K_{c}$, then by lemma $8, r^{-1} K_{a} r=K_{b}$ and $r^{-1} K_{a} r=K_{a}$ which is a contradiction.

Lemma 10. If $a, b, c$ are three different elements of $\Sigma$, then $R=K_{a} K_{b} K_{c}$.

Proof. By lemma $6 K_{a} K_{b} \subseteq R$. Thus $K_{a} K_{b} K_{c} \subseteq R K_{c}=R$ by lemma 7 .

If $r \in R$, then by lemma 9 either $r \in K_{a} K_{b} \subseteq K_{a} K_{b} K_{c}$ or $r \in K_{a} K_{c} \subseteq$ $K_{\mathrm{a}} K_{b} K_{c}$.

Hence $R=K_{\mathrm{a}} K_{\mathrm{b}} K_{\mathrm{c}}$.

We can now prove

THEOREM 1. If $G$ is a doubly transitive group on a set $\Sigma$, only the identity fixes three symbols of $\Sigma$, and $G$ contains a sharply doubly transitive subset $R$, then $R$ is a normal subgroup of $G$.

Proof. If $\Sigma$ contains less than three symbols the theorem is obvious.

Suppose $\Sigma$ contains three or more symbols and that $a, b, c \in \Sigma$ are all different.

Then, by lemma $10, R=K_{a} K_{b} K_{c}$. Therefore

$$
\begin{aligned}
R^{2} & =K_{a} K_{b} K_{c} K_{a} K_{b} K_{c} \\
& \cong R K_{c} K_{a} K_{b} K_{c} \text { by lemma } 6 \\
& =R \text { by repeated application of lemma } 7 .
\end{aligned}
$$

This proves the theorem.

The following application to projective planes is noted.

THEOREM 2. Let $R$ be a ternary ring of a projective plane of order $n$. Then the elements of $R$ may be regarded as a set of permutations on a set $\Sigma$ of $n$ elements and as such they form a sharply doubly transitive set. Let $G(R)$ se the permutation group generated by $R$. Then either $G(R)=R$ or $G(R)$ :ontains a permutation, not the identity, which fixes three symbols of $\Sigma$.

\section{References}

1] W. Feit, 'On a class of doubly transitive permutation groups', Illinois J. Math. 4 (1960), $170-186$.

2] N. Itô, 'On a class of doubly transitive permutation groups', Illinois J. Math. 6 (1962), $341-352$.

3] M. Suzuki, 'On a class of doubly transitive groups', Ann. of Math. 75 (1962), 105-145.

Iniversity of Auckland .uckland, New Zealand 